\title{
Subunit composition of respiratory chain complex 1 and its responses to oxygen in mitochondria from human donor livers
}

\author{
S. E. Khorsandi ${ }^{1} \mathbb{D}$, J. W. Taanman ${ }^{2}$ and N. Heaton ${ }^{1 *}$
}

\begin{abstract}
Objective: Donor liver function in transplantation is defined by mitochondrial function and the ability of mitochondria to recover from the sequence of warm and/or cold ischemia. Mitochondrial resilience maybe related to assembly and- subunit composition of Complex 1. The aim of this study was to determine if Complex 1 subunit composition was different in donor livers of varying quality and whether oxygen exposure had any effect.

Results: Five human livers not suitable for transplant were split. One half placed in cold static storage and the other half exposed to $40 \%$ oxygen for $2 \mathrm{~h}$. Protein was extracted for western blot. Membranes were probed with antibodies against $\beta$-actin and the following subunits of Complex 1: MTND1, NDUFA10, NDUFB6 and NDUFV2. No difference in steady state Complex 1 subunit composition was demonstrated between donor livers of varying quality, in terms of steatosis or mode of donation. Neither did exposure to oxygen influence Complex 1 subunit composition. This small observational study on subunit levels suggest that Complex 1 is fully assembled as no degradation of subunits associated with the different parts of the enzyme was seen.
\end{abstract}

Keywords: Mitochondria, Liver transplantation, Complex 1, Oxygen, Donor after cardiac death

\section{Introduction}

Liver transplantation is limited by the lack of organs and combined with lengthening waiting lists results in a waiting list mortality of up to $20 \%$. To address this disparity the marginal liver in terms of donor age, liver steatosis or donation after cardiac death (DCD) is increasingly used. But there is a risk to the recipient in terms of graft dysfunction, graft loss or death.

Previous work has demonstrated that mitochondrial recovery and ability to generate adenosine triphosphate (ATP) effectively is a determinant of survival after liver transplantation. Mitochondria are central to cellular health through the production of ATP by oxidative phosphorylation (OXPHOS) and reactive oxygen species (ROS) that both drive and define cell function [1]. Consequently, many of the strategies used to optimize

\footnotetext{
*Correspondence: nigel.heaton@nhs.net

1 Institute of Liver Studies, King's College Hospital, Kings College London, London SE5 9RS, UK

Full list of author information is available at the end of the article
}

the donor liver are aiming to limit mitochondrial injury, such as solutions for cold static storage and minimizing ischemic times (warm and cold).

The largest complex of the respiratory chain is Complex 1 (> $900 \mathrm{kDa})$. It is an L-shaped multimeric protein complex, composed of 45 subunits and is the electron entry point of the respiratory chain. Disruption in the organization of Complex 1 can lead to an energy switch from OXPHOS to glycolysis [2]. The factors that drive the formation of mitochondrial respirasomes, the functional units of respiration, composed of complex 1, 3 and 4 remain unknown, but Complex 1 assembly and activation is regarded as a critical event. Potentially, donor liver function could be defined by mitochondrial resilience, as determined by assembly and subunit composition of Complex 1.

The aim of this work was to determine if Complex 1 subunit composition in discarded human donor livers of varying quality was different, and whether composition could be modulated by oxygen exposure. 


\section{Main text \\ Methods \\ Donor human livers and the split liver model}

Donor livers $(n=5)$ utilized in this study had been declined for both solid organ and cell transplantation. Table 1 summarizes the clinical characteristics of the donor livers used and reason for discard. The two donor liver types that were used in this study were: (1) donor after brainstem death (DBD) and (2) donor after cardiac death (DCD). In both donor types, dual aortic and portal perfusion was performed using University of Wisconsin Solution (UW) at $4{ }^{\circ} \mathrm{C}$. In the DBD liver, organ perfusion with blood was maintained throughout the preparatory donor dissection up to cannulation. Whereas in the DCD liver, aortic and portal vein cannulation was performed after circulatory arrest. The warm ischemic time (WIT), which is unique to the DCD liver, was defined from which agonal observation occurred first, either a systolic of $50 \mathrm{mmHg}$ or oxygen saturation of $70 \%$, to aortic cannulation. The cold ischemic time (CIT) was defined as the time from the start of cold aortic perfusion to the start of oxygen exposure i.e. anterograde persufflation (A-PSF).

To assess the response of the donor liver to A-PSF an experimental split model was developed. This involved performing a surgical left lateral segment (LLS) split. Producing two sections of liver for study from one donor liver with an inflow portal vein (PV) and outflow hepatic vein. A-PSF was via the PV with gaseous venting through the hepatic veins. The experimental $\left(\mathrm{O}_{2}\right.$ exposed/A-PSF) section of donor liver was kept on ice in UW throughout, while the non-PSF or control section of donor liver was rebagged for standard cold static storage in UW on ice.

\section{Electrochemical oxygen concentrator and anterograde persufflation}

Oxygen enriched atmospheric air was supplied with a portable electrochemical oxygen concentrator (Giner
Inc, Newton, MA, USA). For this study atmospheric air was enriched to $40 \%$ oxygen. The PV was cannulated and A-PSF was performed with $40 \% \mathrm{O}_{2}$ via the PV at a manifold pressure of $15-20 \mathrm{mmHg}$ to achieve flows of $15-20 \mathrm{ml} / \mathrm{min}$. Persufflation was performed for $2 \mathrm{~h}$. Trucut biopsies were then taken from both sides of the liver (A-PSF/experimental $\mathrm{O}_{2}$ exposed and non-PSF/ control) for formalin fixation and snap freezing in liquid nitrogen for storage at $-80^{\circ} \mathrm{C}$.

\section{Protein extraction and analysis}

Frozen liver biopsies were homogenized on ice using a micropestle in a $1.5 \mathrm{ml}$ microcentrifuge tube. Homogenization buffer composition is as follows: $10 \mathrm{mM}$ Hepes $\mathrm{NaOH} \mathrm{pH}$ 7.4, $1 \mathrm{mM}$ sodium EDTA, $250 \mathrm{mM}$ sucrose, $1 \mu \mathrm{g} / \mathrm{ml}$ pepstatin $\mathrm{A}, 1 \mu \mathrm{g} / \mathrm{ml}$ leupeptin and $1 \mathrm{mmol}$ phenylmethanesulfonyl fluoride. After homogenization the samples were stored at $-80{ }^{\circ} \mathrm{C}$ for later use. Homogenates were further extracted in homogenization buffer containing $1.5 \%$ lauryl $n$-dodecyl $\beta$-D-maltoside (Anatrace, USA) on ice. All reagents were from SigmaAldrich unless otherwise stipulated. After extraction, samples were centrifuged at $13,000 \mathrm{~g}$ for $10 \mathrm{~min}$ at $4{ }^{\circ} \mathrm{C}$. The cytoplasmic fraction (supernatant) was transferred into pre-cooled new tubes. For quantitation of total protein the Pierce ${ }^{\mathrm{TM}}$ BCA Protein Assay Kit (Thermo Scientific, USA) was used.

For protein electrophoresis and blotting the miniPROTEAN $^{\circledR}$ Tetra Vertical Electrophoresis Cell, 4-15\% mini-PROTEAN ${ }^{\circledR}$ TGX Stain-Free ${ }^{\text {TM }}$ Precast Gels and Trans-Blot $^{\circledR}$ Turbo $^{\mathrm{TM}}$ Transfer System (Bio-Rad Lab Ltd, UK) were used. Reference ladder was Precision Plus Protein $^{\text {TM }}$ Dual Color Standards (Bio-Rad Ltd, UK). $20 \mu \mathrm{g}$ of protein was loaded per lane. The polyvinylidene difluoride (PVDF) membrane was dried overnight before blocking and probing. In brief, membranes were blocked in 5\% skimmed milk/phosphate buffered saline, $0.3 \%$

Table 1 Summary of the clinical characteristics of the donor liver studied

\begin{tabular}{llllll}
\hline & Liver $\mathbf{1}$ & Liver $\mathbf{2}$ & Liver $\mathbf{3}$ & Liver & Liver $\mathbf{5}$ \\
\hline Donor age (years) & 47 & 60 & 49 & 55 & 59 \\
Graft type & DBD & DCD & DCD & DCD & DBD \\
Steatosis & $50 \%$ & $0 \%$ & $30 \%$ & $20 \%$ & NA \\
WIT (min) & NA & 24 & 15 & 20 & 10 \\
CIT (h) & 8 & 12 & 12 & 10 & \\
Reason for clinical discard & Steatohepatitis with fibrosis & Perfusion concern & Steatosis & Lymphoproliferative disorder Cholangiocarcinoma concern
\end{tabular}

Graft types studied included donor after brain stem death (DBD) and donor after cardiac death (DCD). Liver steatosis is expressed as a percentage (\%) based on histological assessment of the liver using Hematoxylin and Eosin stain. The warm ischemic time (WIT) is only applicable to the DCD liver and is from the time of oxygen saturation of $70 \%$ or systolic blood pressure of $50 \mathrm{mmHg}$ in the donor to the time of aortic cannulation in the donor. The cold ischemic time (CIT) for this study is from the time of aortic cannulation in the donor to the time when anterograde persufflation $\left(\mathrm{O}_{2}\right.$ exposure) starts. NA Not applicable 
Tween (PBS-T) for $1 \mathrm{~h}$ followed by rinsing in PBS-T. The membrane was then probed with the primary antibody in PBS-T for $2 \mathrm{~h}$ at room temperature unless otherwise stated.

Primary antibodies used and dilutions are as follows: $\beta$-actin, $42 \mathrm{kDa}, 1: 120,000$ (abcam ${ }^{\circledR}$ ab6276); NDUFB6 (distal part of membrane arm), $17 \mathrm{kDa}, 1: 1500$ overnight incubation at $4{ }^{\circ} \mathrm{C}\left(\right.$ abcam $^{\circledR}$ ab110244); NDUFV2 (distal part of matrix arm), $24 \mathrm{kDa}, 1: 1000$ overnight incubation at $4{ }^{\circ} \mathrm{C}$ (proteintech ${ }^{\mathrm{TM}} 15301-1-\mathrm{AP}$ ); NDUFA10 (middle part of membrane arm), $42 \mathrm{kDa}\left(\right.$ abcam $^{\circledR}$ ab174829); and MTND1 (proximal part of membrane arm), $36 \mathrm{kDa}$ (proteintech $^{\text {TM }}$ 19703-1-AP).

Appropriate secondary antibodies (Dako Agilent Pathology Solutions polyclonal goat anti-mouseP026002-2 or anti-rabbit IgG-HRP-P044801-2) were used, diluted 1:3000 in PBS-T and incubated with the membrane for $1 \mathrm{~h}$. Clarity ${ }^{\mathrm{TM}}$ Western ECL Substrate (BioRad Lab Ltd, UK) was used to develop the image. Image capture, background subtraction and quantification of band signal intensity was performed using Image $\mathrm{Lab}^{\mathrm{TM}}$ Software v5.1 (Bio-Rad Lab Ltd, UK). The protein of interest was normalized to $\beta$-actin. Human nomenclature for Complex 1 subunits has been used.

\section{Statistics}

Where appropriate, data is expressed as a mean and standard deviation. For comparison of groups the unpaired two tailed $t$ test was used, significance value was set as $\mathrm{p}<0.05$. Statistical analysis was performed with GraphPad Prism v7.0b (GraphPad Software, Inc. USA).

\section{Results}

\section{Donor liver demographics}

Five human donor livers declined for transplantation were split, considering graft type, 2 were DBD and 3 DCD, producing 10 sections of liver for study. The mean donor age was $54 \pm 5.8$ years, cold ischemic time $10.4 \pm 1.7 \mathrm{~h}$ and warm ischemic time, where applicable, was $19.7 \pm 4.5 \mathrm{~min}$. On Hematoxylin and Eosin histology, steatosis was not demonstrated in 2 livers and in the remaining 3 , steatosis ranged from 20 to $50 \%$. The main documented reason for donor liver decline for transplantation was pathology in the donor, identified histologically as steatosis in 2 and cancer risk in another 2 . In the remaining declined liver, there were concerns about preservation perfusion (Table 1). There were no technical issues on splitting the liver or on using A-PSF. In all cases, persufflation via the PV was successful, with minimal air leak from the cut surface and effective venting of $\mathrm{O}_{2}$ via the hepatic vein.

\section{Complex 1 subunit protein analysis}

Figure 1 shows the western blot images for $\beta$-actin (housekeeping/reference protein) and the following subunits of Complex 1: MTND1 (proximal part of membrane arm), NDUFA10 (middle part of membrane arm), NDUFB6 (distal part of membrane arm) and NDUFV2 (distal part of matrix arm). On comparison of studied subunits according to liver section graft type, DBD $(\mathrm{n}=4)$ versus DCD $(\mathrm{n}=6)$ there was no difference in protein expression (NDUFB6, $\mathrm{p}=0.53$; NDUFV2, $\mathrm{p}=0.72$; NDUFA10, $\mathrm{p}=0.75 ;$ MTND1, $\mathrm{p}=0.48$; Fig. 2a). Similarly, no difference in subunit composition was seen when comparing the steatotic $(n=6)$ to non steatotic $(\mathrm{n}=4)$ donor liver section (NDUFB6, $\mathrm{p}=0.81$; NDUFV2, $\mathrm{p}=0.59 ;$ NDUFA10, $\mathrm{p}=0.89 ;$ MTND1 $\mathrm{p}=0.28$; Fig. $2 \mathrm{~b}$ ). When the effect of oxygen produced by A-PSF was analyzed, again no difference in subunit composition in Complex 1 was demonstrated on comparison of $\mathrm{O}_{2}$ exposed $(\mathrm{n}=5)$ to non $\mathrm{O}_{2}(\mathrm{n}=5)$ exposed liver sections (NDUFB6, $\mathrm{p}=0.36$; NDUFV2, $\mathrm{p}=0.37$; NDUFA10, $\mathrm{p}=0.93$; MTND1, $\mathrm{p}=0.52$; Fig. 2c).

\section{Discussion}

In this small observational study, no changes in Complex 1 subunit levels was demonstrated in the donor liver on exposure to oxygen when used as a resuscitative strategy after cold static storage. Suggesting that Complex 1 is fully assembled, as no degradation of subunits associated with the two arms was seen. Additionally, neither steatosis or graft type (DBD v DCD) had any bearing on Complex 1 subunit composition.

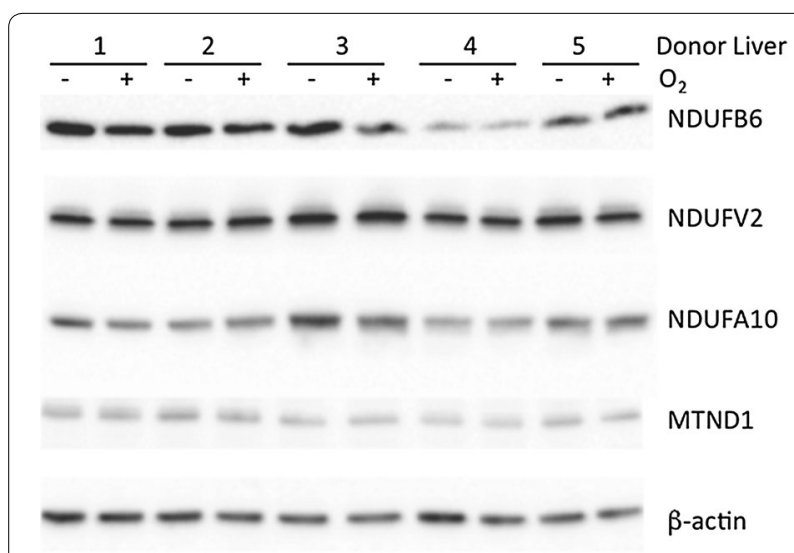

Fig. 1 Western blot for the subunits of Complex 1. Subunits analyzed were NDUFB6 (distal part of membrane arm), NDUFV2 (distal part of matrix arm), NDUFA10 (middle part of membrane arm), MTND1 (proximal part of membrane arm) and the housekeeping $\beta$-actin protein 
Fig. 2 a Graphical summary of changes in Complex 1 subunits of the liver in donor after brainstem death $v$ donor after cardiac death. Changes in levels of Complex 1 subunits are expressed as a ratio to $\beta$-actin comparing expression level of given subunit in donor after brainstem death ( $\mathrm{DBD}, \mathrm{n}=4)$ compared to donor after cardiac death $(D C D, n=6)$ liver section. Complex 1 subunits compared were NDUFB6 (distal part of membrane arm), NDUFV2 (distal part of matrix arm), NDUFA10 (middle part of membrane arm) and MTND1 (proximal part of membrane arm). Y-axes represent given Complex 1 subunit signal relative to $\beta$-actin signal. Error bars indicate standard deviation. No significant difference was demonstrated. b Graphical summary of changes in Complex 1 subunits steatotic v non steatotic liver. Changes in levels of Complex 1 subunits are expressed as a ratio to $\beta$-actin comparing expression level of given subunit in the non steatotic $(n=4)$ compared to steatotic $(n=6)$ donor liver section. Complex 1 subunits compared were NDUFB6 (distal part of membrane arm), NDUFV2 (distal part of matrix arm), NDUFA10 (middle part of membrane arm) and MTND1 (proximal part of membrane arm). Y-axes represent given Complex 1 subunit signal relative to $\beta$-actin signal. Error bars indicate standard deviation. No significant difference was demonstrated. c Graphical summary of changes in Complex 1 subunits $\mathrm{O}_{2} \vee$ non $\mathrm{O}_{2}$ exposed section of liver. Changes in levels of Complex 1 subunits are expressed as a ratio to $\beta$-actin comparing expression level of given subunit in the section of liver exposed to oxygen $\left(\mathrm{O}_{2}\right)$ via anterograde persufflation $(n=5)$ compared to the control section of liver subjected to cold static storage $(n=5)$. Complex 1 subunits compared were NDUFB6 (distal part of membrane arm), NDUFV2 (distal part of matrix arm), NDUFA10 (middle part of membrane arm) and MTND1 (proximal part of membrane arm). Y-axes represent given Complex 1 subunit signal relative to $\beta$-actin signal. Error bars indicate standard deviation. No significant difference was demonstrated

Complex 1 is a L-shaped structure composed of 45 subunits. One limb is a hydrophobic membrane arm sitting in the mitochondrial inner membrane and the other arm is hydrophilic, projecting into the mitochondrial matrix [2]. Complex 1 has three functional modules [3]: the $\mathrm{N}$ module electron accepting (input) and the $\mathrm{Q}$ module ubiquinone reducing (electron output) both located in the hydrophilic arm and the P proton pumping/translocase within the hydrophobic membrane arm. The energy generated by electron transfer within the matrix arm is transduced, by conformational changes in the membrane arm, to pump four protons into the mitochondrial intermembrane space.

Supercomplex assembly is thought to provide structural and functional advantages to the enzymes of the respiratory chain [4], thereby improving efficiency of the respiratory chain production of ATP, as well as reducing ROS generation [5-7]. More recent work suggests that the respiratory enzymes exist in a solid complex of varying ratios of Complex 1, 3 and 4 to form the supercomplex [8].

Low energy charge and purine quantity in donor liver parenchyma determines poor outcome in transplantation, which is related to mitochondrial function. The application of persufflation, gaseous perfusion with

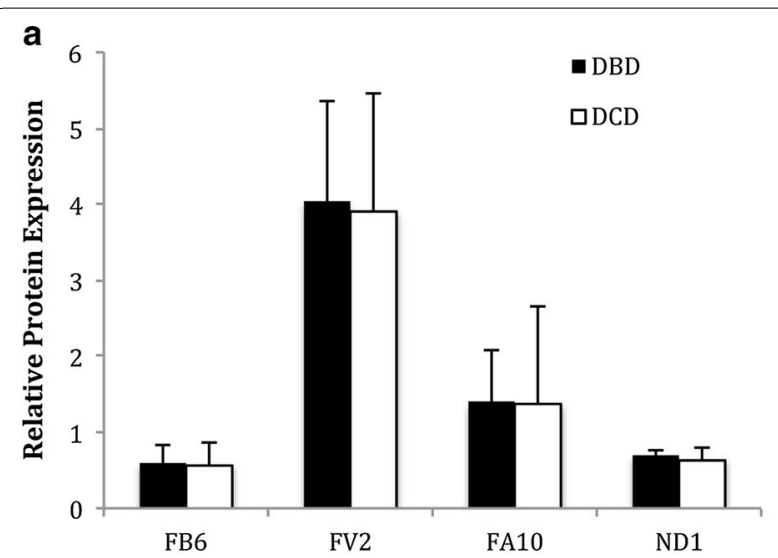

b
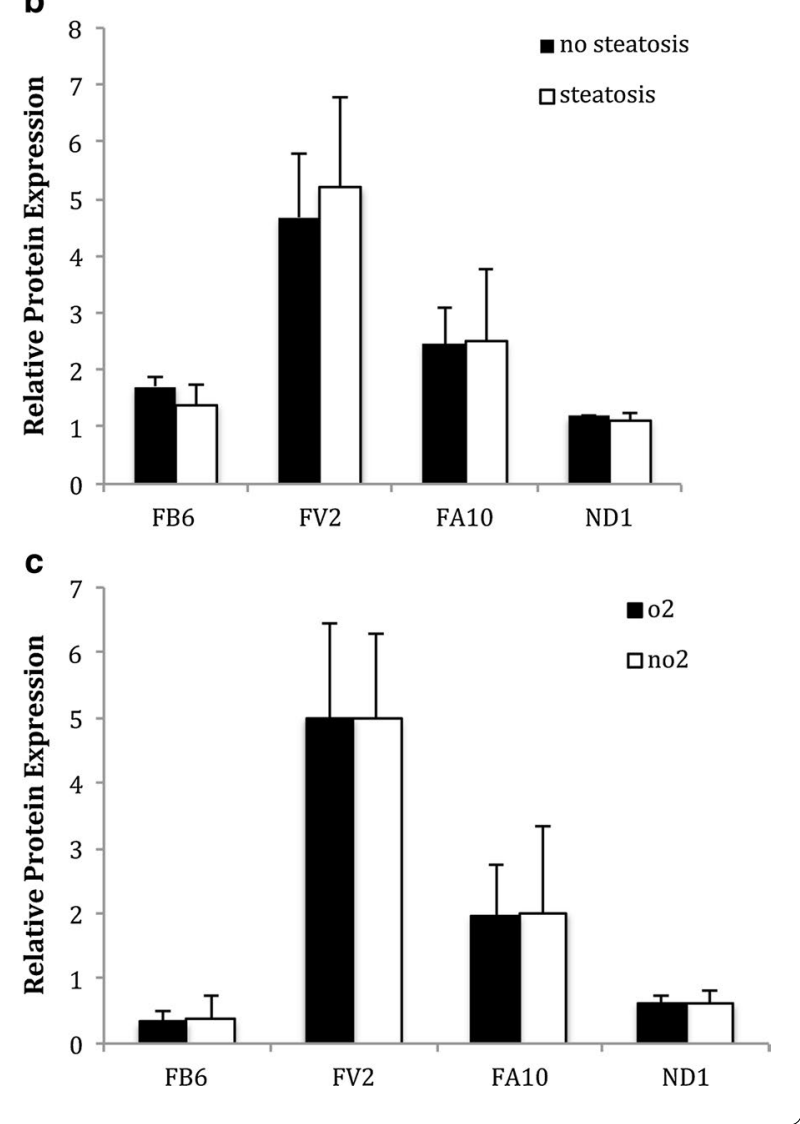

oxygen, to 'resuscitate' organs for transplantation has been explored in a number of solid organs [9]. The mitochondrial basis of liver transplant outcome has not been fully elucidated. However, there is some data that shows preservation of Complex 1 is important and improves the quality of cryopreserved human hepatocytes [10]. Additionally, in animal models, Complex 1 activity has been demonstrated to be reduced in both steatosis and increasing CIT [11, 12].

The factors that determine supercomplex assembly and function are still being resolved but it is recognized that 
mitochondrial metabolic needs, as defined by cell type and physiological status are a determinant [13]. Putative assembly factors for the supercomplexes are being characterized [14]. Of interest and relevant to transplantation, a group of hypoxia induced gene products present in the inner mitochondrial membrane have been found to associate with the supercomplex and contribute to its stability/assembly. These hypoxia assembly factors influence supercomplex levels only, with no effect on individual complex levels and maybe the basis for changing supercomplex organization with oxygen availability [15, 16]. This observation could explain the present finding that no change in subunit composition of Complex 1 was seen on exposure to oxygen.

In conclusion, the presented data on subunit composition of Complex 1 suggest it is fully assembled, as no degradation of subunits associated with the different parts of the enzyme was seen. However, more work is needed to characterize, the factors that define the active/de-active state of Complex 1 and whether Complex 1 assembly, is a critical determinant of donor liver mitochondrial function and transplant outcome.

\section{Limitations}

The number of samples available for analysis and comparison are small. No functional assays have been performed to assess Complex 1 activity. Neither have any co-immunoprecipitation experiments been undertaken to demonstrate protein associations. Ideally, Blue Native polyacrylamide gel electrophoresis (BN-PAGE) to study assembly of Complex 1 combined with in gel measurement of enzyme activity on a larger number of transplanted and non transplanted livers needs to be undertaken.

\section{Abbreviations \\ ATP: adenosine triphosphate; A-PSF: anterograde persufflation; DBD: donor after brainstem death; DCD: donor after cardiac death; ETC: electron transport chain; LM: lauryl n-dodecyl $\beta$-D-maltoside; LLS: left lateral segment; MTNDI: mitochondrial encoded NADH dehydrogenase I; NDUFB6: NADH dehydroge- nase (Complex 1) 1ß subcomplex 6; NDUFA10: NADH dehydrogenase (ubiqui- none) 1a subcomplex 10; NDUFV2: NADH dehydrogenase (ubiquinone) flavoprotein 2; PBS-T: phosphate buffered saline-0.3\% Tween; OXPHOS: oxidative phosphorylation; PVDF: polyvinylidene difluoride; PV: portal vein; ROS: reactive oxygen species; UW: University of Wisconsin Solution; WIT: warm ischemic time.}

\section{Authors' contributions}

SEK, NH and JWT study concept. SEK and JWT designed and performed study. SEK collected and analyzed data. SEK, JWT and NH wrote the paper. All authors read and approved the final manuscript.

\section{Author details}

${ }^{1}$ Institute of Liver Studies, King's College Hospital, Kings College London, London SE5 9RS, UK. ${ }^{2}$ Department of Clinical Neurosciences, Institute of Neurology, University College London, London, UK.

\section{Competing interests}

The authors declare that have no competing interests.

\section{Acknowledgements}

The specialist nurses in organ donation.

\section{Availability of data and materials}

The data generated from the current study is available from the corresponding author on reasonable request.

\section{Consent for publication}

Not applicable. No identifiable patient (donor) data has been presented.

\section{Ethics approval and consent to participate}

Research undertaken had full institutional ethical approval (Research Ethics Committee (REC 01-106), National Research Ethics Committee (NRES) London-Dulwich, UK). Written consent for research on the donor liver was obtained from the donor family at the same time that consent was taken for organ donation by the Specialist Nurse in Organ Donation.

Funding

There is no funding to declare.

\section{Publisher's Note}

Springer Nature remains neutral with regard to jurisdictional claims in published maps and institutional affiliations.

Received: 5 July 2017 Accepted: 24 October 2017

Published online: 02 November 2017

\section{References}

1. Nunnari J, Suomalainen A. Mitochondria: in sickness and in health. Cell. 2012;148(6):1145-59.

2. Zhu J, Vinothkumar KR, Hirst J. Structure of mammalian respiratory complex I. Nature. 2016;536(7616):354-8.

3. Fassone E, Rahman S. Complex I deficiency: clinical features, biochemistry and molecular genetics. J Med Genet. 2012;49:578-90.

4. Lenaz G, Genova ML. Structure and organization of mitochondrial respiratory complexes: a new understanding of an old subject. Antioxid Redox Signal. 2010;12:961-1008.

5. Dudkina NV, Eubel H, Keegstra W, Boekema EJ, Braun HP. Structure of a mitochondrial supercomplex formed by respiratory-chain complexes I and III. PNAS. 2005:102:3225-9.

6. Rosca MG, Vazquez EJ, Kerner J, Parland W, Chandler MP, Stanley W, Sabbah HN, Hoppel CL. Cardiac mitochondria in heart failure: decrease in respirasomes and oxidative phosphorylation. Cardiovasc Res. 2008:80:30-9.

7. Gómez LA, Monette JS, Chavez JD, Maier CS, Hagen TM. Supercomplexes of the mitochondrial electron transport chain decline in the aging rat heart. Arch Biochem Biophys. 2009;490:30-5

8. Vartak R, Porras CA, Bai Y. Respiratory supercomplexes: structure, function and assembly. Protein Cell. 2013;4(8):582-90.

9. Suszynski TM, Rizzari MD, Scott WE III, Tempelman LA, Taylor MJ, Papas KK. Persufflation (or gaseous oxygen perfusion) as a method of organ preservation. Cryobiology. 2012;64:125-43.

10. Stéphenne X, Najimi M, Ngoc DK, Smets F, Hue L, Guigas B, Sokal EM. Cryopreservation of human hepatocytes alters the mitochondrial respiratory chain complex 1. Cell Transplant. 2007;16(4):409-19.

11. Chu MJ, Premkumar R, Hickey AJ, Jiang Y, Delahunt B, Phillips AR, Bartlett AS. Steatotic livers are susceptible to normothermic ischemia-reperfusion injury from mitochondrial Complex-I dysfunction. World J Gastroenterol. 2016;22(19):4673-84.

12. Chu MJ, Hickey AJ, Jiang Y, Petzer A, Bartlett AS, Phillips AR. Mitochondrial dysfunction in steatotic rat livers occurs because a defect in complex I makes the liver susceptible to prolonged cold ischemia. Liver Transplant. 2015;21(3):396-407

13. Bianchi C, Genova ML, Castelli GP, Lenaz G. The mitochondrial respiratory chain is partially organized in a supercomplex assembly: kinetic evidence using flux control analysis. J Biol Chem. 2004;279:36562-9. 
14. McKenzie M, Ryan MT. Assembly factors of human mitochondrial complex I and their defects in disease. IUBMB Life. 2010;62:497-502.

15. Wang J, Cao Y, Chen Y, Chen Y, Gardner P, Steiner DF. Pancreatic $\beta$ cells lack a low glucose and $\mathrm{O}_{2}$-inducible mitochondrial protein that augments cell survival. PNAS. 2006;103:10636-41.
16. Chen YC, Taylor EB, Dephoure N, Heo JM, Tonhato A, Papandreou I, Nath N, Denko NC, Gygi SP, Rutter J. Identification of a protein mediating respiratory supercomplex stability. Cell Metab. 2012;15:348-60.

\section{Submit your next manuscript to BioMed Central} and we will help you at every step:

- We accept pre-submission inquiries

- Our selector tool helps you to find the most relevant journal

- We provide round the clock customer support

- Convenient online submission

- Thorough peer review

- Inclusion in PubMed and all major indexing services

- Maximum visibility for your research

Submit your manuscript at www.biomedcentral com/submit
() Biomed Central 